\title{
2000-2001 Canadian Association of Emergency Physicians Research Grant Competition
}

\author{
Brian H. Rowe, MD
}

$\mathrm{T}$ he Canadian Association of Emergency Physicians (CAEP) Research Grants Competition is an example of supplemental research funding developed by CAEP that is only made available to researchers in Emergency Medicine. Over the years, this competition has allowed CAEP researchers and their collaborators to compete for small grants (up to $\$ 5,000)$ to conduct research relevant to Emergency Medicine. Several previous CAEP grant recipients presented their research results at the CAEP Conference in Saint John, NB, in June 2000. These grants are made possible by the generosity and commitment of Hoffman-La Roche, the annual sponsor of this competition. HoffmannLa Roche has been a major contributor and supporter of Emergency Medicine research in Canada and they are the sole contributor to this research collaboration.

This grant competition was held this year for the fourth time in 5 years. The CAEP Research Grants Competition was able to secure a $\$ 25,000$ unrestricted educational grant from HoffmannLa Roche to support the 2000-2001 competition. Consequently, we were able to accommodate all research applications again this year.

We are pleased to announce the results of the CAEP Research Grants Competition for 2000-2001. The following list outlines the name and affiliation of the principal investigator, the proposal title of the project, and the

Associate Professor, University of Alberta; CAEP Research Chair for the CAEP

Research Committee funding allocation from CAEP to each recipient.

1. Atul Kapur, MD. Division of Emergency Medicine, University of Ottawa, Ottawa, Ont. Emergency department treatment of atrial fibrillation. $\$ 5,000$.

2. Mark Yarema, MD. Division of Emergency Medicine, University of Calgary, Calgary, Alta. A comparison of the effectiveness of the 20 hour intravenous versus the 72 hour oral n-acetylcysteine protocols in the treatment of acute acetaminophen overdose. $\$ 4,919.50$

3. Jeff Perry, MD. Division of Emergency Medicine, University of Ottawa, Ottawa, Ont. Are intubating conditions created during rapid sequence induction intubation using rocuronium comparable to succinylcholine? A meta analysis. $\$ 800$.

4. David Petrie, MD. Department of Emergency Medicine, Dalhousie University and Queen Elizabeth II HSC. Do outcomes improve after integrating the EMS system in the follow-up care of discharged CHF patients? \$5,000.

5. Martin Pusic, MD. Montreal Children's Hospital, Pediatric Emergency Department, McGill University, Montreal, Que. Predictors of children at risk for negative sequelae following concussion. $\$ 5,000$.
In accepting the research award, certain requirements were made of the primary investigators. 1) All grants were provided to recipients as peer-reviewed unrestricted educational grants from CAEP; in other words, all budgets must be expended on grant items and not on institutional overhead. 2) It is expected that grant recipients will provide CAEP, through the Research Committee, with a final report and a full financial statement regarding the allocation of resources at the conclusion of the study. 3) A completed manuscript is required prior to reapplying for another CAEP grant. 4) Finally, all presentations and publications of the findings are required to acknowledge the CAEP Research Grant contributions to their research.

On behalf of the Research Committee, the CAEP Board and HoffmannLa Roche, we thank all CAEP members who took the time to apply for these grants. We congratulate the grant recipients for their success in this year's competition. We wish them success in their research endeavours and hope to see the results presented at a future CAEP meeting.

Acknowledgements: The CAEP Research Committee acknowledges the support of HoffmannLa Roche in making these grants available this year. In particular, we recognize Alan LeVogeur (Product Manager, Activase) and Dr. Lorraine Paul (Associate Director, Cardiovascular) of HoffmannLa Roche, for their interest and commitment to this project. We thank Mrs. Sue Norrington for her efforts in coordinating the competition and Dr. Douglas Sinclair for his efforts in promoting this collaboration.

Correspondence to: Dr. Brian Rowe; Brian.Rowe @ ualberta.ca 